\title{
Modulatable optical radiators and metasurfaces based on quantum nanoantennas
}

\author{
Pai-Yen Chen ${ }^{1, *}$ and Mohamed Farhat ${ }^{2}$ \\ ${ }^{1}$ Department of Electrical and Computer Engineering, Wayne State University, Detroit, Michigan 48202, USA \\ ${ }^{2}$ Division of Computer, Electrical, and Mathematical Sciences and Engineering, \\ King Abdullah University of Science and Technology (KAUST), Thuwal 23955-6900, Saudi Arabia \\ (Received 11 October 2014; revised manuscript received 22 December 2014; published 20 January 2015)
}

\begin{abstract}
We investigate the tunable and switchable optical radiators and metamaterials formed by metallic nanodipole antennas with submicroscopic gaps $(1.2 \mathrm{~nm})$, of which linear and third-order nonlinear quantum conductivities are observed due to the photon-assisted tunneling effect. The quantum conductivities induced at the nanogap are relevant to power dissipations, which can be enhanced by the strongly localized optical fields associated with the plasmonic resonance. We demonstrate that the scattering property of an individual quantum nanoantenna and the transparency of a metamasurface constituted of it can be tuned by electrostatically controlling the linear conductivity (electronic tuning) or by adjusting the irradiation intensity that varies the nonlinear quantum conductivity (all-optical tuning).
\end{abstract}

DOI: 10.1103/PhysRevB.91.035426

PACS number(s): 73.20.Mf, 78.67.-n, 42.50.-p

Optical nanoantennas consisting of single or paired metallic nanoparticles provide an effective route to couple the propagating light to and from deeply subwavelength volumes [1-9]. When metallic nanoparticles are irradiated by light, the coherent and collective oscillations of conduction band-free electrons (surface plasmon) can induce large surface electric fields. This strong light-matter interaction mediated by surface plasmon in metallic nanostructures has been shown to localize optical fields to a subdiffraction-limited region, enhancing significantly the excitation and emission of quantum emitters, i.e., single molecule or quantum dot [1-9], and offering the flexible control of nanofocused radiation directionality [10-14] and the promising route for wireless interconnections between ultracompact photonic devices [15]. Recent advances in nanofabrication techniques allow the precise patterning of individual and arrays of nanoantennas through nanolithography. Although the individual response of a nanoantenna provides various exciting possibilities, its scattering cross section associated with total scattered power is typically very minimal due to its extremely small size. For this reason, nanoantenna experiments are usually demonstrated with the two-dimensional (2-D) planar array of nanoantennas, representing a type of optical metasurface or metamaterial with custom-designed optical properties for imaging and holography [16-18], optical switching [14,19,20], polarization and phase controlling [10,11,21,22], energy harvesting [2327], and nonlinear optical [14,28-30] applications.

Different from their RF and microwave counterparts, the peculiar longitudinal electric field distribution inside the optical metallic nanodipole antenna is responsible for the flow of the optical displacement current along the nanoantenna and the peculiar polarization of illumination. The interaction between optical fields and displacement currents of nanoscale objects can be described by the optical nanocircuits theorem [5,31], which has been theoretically and experimentally investigated by several groups in terms of the concepts of tuning, loading, and matching optical nanoantennas with small

*Corresponding author: pychen@wayne.edu dielectric (capacitive), metallic (inductive), and nonlinear material nanoparticles as useful lumped elements in the optical regime $[14,19,32,33]$. The optical nanocircuits within the realm of classical electromagnetism can perfectly explain the physical phenomena of nanoantennas, with its minimum dimensions greater than a few nanometers. However, when the minimum feature size is reduced to less than a few nanometers, the quantum mechanical effect emerges inevitably, bringing new physical phenomena not observed in the classic treatment. For instance, if the gap between two metallic dipole arms of nanoantenna is sufficiently small, the electron tunneling can be driven by electromagnetic fields [34-38], resulting in the frequency-dependent quantum conductivity that would intrinsically limit the field enhancement at the nanogap [39-43]. Such a result is very different from the classical nanocircuit model that predicts an ever-increasing local field enhancement when the gap is squeezed. In addition, in the quantum mechanical model, a third-order nonlinear quantum conductivity related to the two-photon absorption may exist when the nanoantenna is illuminated by an intense pulsed laser [39-43].

In this paper, we will investigate the modulatable and switchable optical radiators, metasurfaces, and metamaterials, which are formed by individual or arrayed nanodipole antennas with a submicroscopic gap, within the quantum realm of photon-assisted electron (plasmon) tunneling [35-37]. The overarching goal of this work is to understand the optical tunability enabled by electronically and optically variable quantum conductivities existing in specific nanodipole antennas. Different from previous efforts $[14,19,20]$, where optical nonlinearities are introduced by loading nanoparticles with nonlinear susceptibilities, the proposed modulatable nanoantennas and metamaterials are enabled by the quantum conductivities, intrinsically induced by the charge exchanges in the submicroscopic air gap between two metal regions of the nanodipole [see Figs. 1(a) and 1(b)], so called quantum nanoantennas. Further, such quantum conductivities can be electronically tuned by electrostatic biasing, as shown in Fig. 1(a), or optically tuned by adjusting the intensity of illumination. Here the patterned graphene electrodes, being transparent for near-infrared and visible light, are used to 


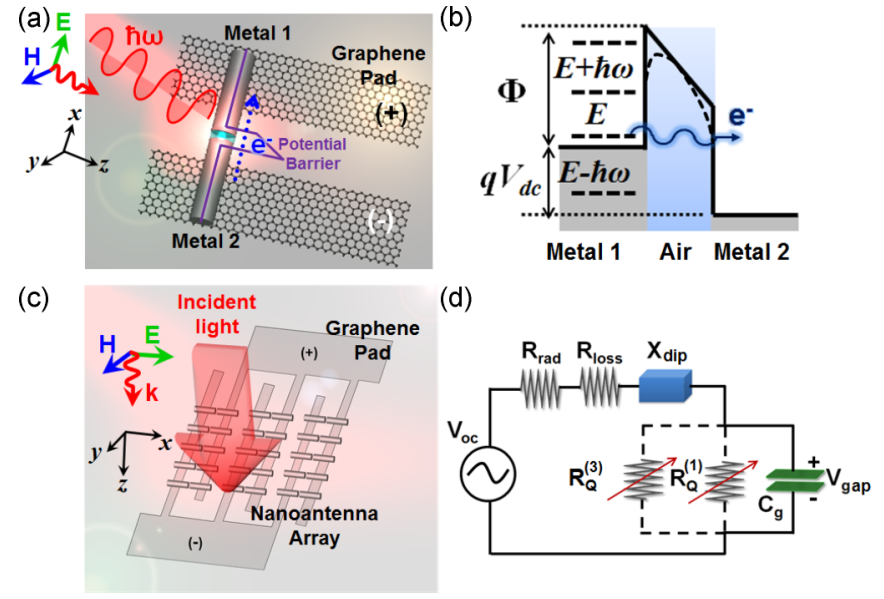

FIG. 1. (Color online) Schematics of a metallic nanoantenna with submicroscopic gap, which makes a MIM (tunneling) nanojunction. (b) Energy band diagram for the electron photon-assisted tunneling process. (c) Modulatable metasurface using biased nanoantennas, where patterned graphene are used as transparent electrodes, with a $\sim 97.7 \%$ transparency at near-infrared and visible frequencies. (d) Nanocircuit model for the nanoantenna in (a); here $C_{g}$ is the geometryyielded nanocapacitor, $R_{Q}^{(1)}$ and $R_{Q}^{(3)}$ are quantum resistances related to the linear and third-order nonlinear quantum conductivities, and the intrinsic dipole impedance $Z_{\mathrm{dip}}=R_{\mathrm{rad}}+R_{\mathrm{loss}}-i X_{\mathrm{dip}}$.

bias the plasmonic nanoantenna [Fig. 1(a)] and metasurface [Fig. 1(c)].

The geometry of the nanodipole antenna interestingly makes a metal-insulator- (air-) metal (MIM) nanojunction, as shown in Fig. 1(a). According to the Simmons mode [34], applying a dc bias $V_{\mathrm{dc}}$, the tunneling current density across the nanojunction can be expressed as

$$
\begin{aligned}
J_{\mathrm{dc}}\left(V_{\mathrm{dc}}\right)= & \frac{4 \pi m_{0} q}{h^{3}} \int_{0}^{\infty} D\left(E_{x}\right) d E_{x} \\
& \times \int_{E_{x}}^{\infty}\left[F(E)-F\left(E+q V_{\mathrm{dc}}\right)\right] d E,
\end{aligned}
$$

where $q$ is the electron charge, $m_{0}$ is the electron mass, $h$ is the Planck constant, $F$ is the Fermi-Dirac distribution function, $E$ and $E_{x}$ are, respectively, the total and longitudinal energy of electrons, and $D$ is the electron transmission probability. The charge transport mechanism in the MIM junction depends on the difference between Fermi levels of two metals (the antenna's dipole arms here). When a dc bias is applied, currents can flow between the two terminals of metallic nanoantennas by means of the tunneling effect, as shown in Fig. 1(b). At equilibrium, the well-known Simmons formula can describe the current-voltage behavior of this MIM junction [34]:

$$
\begin{aligned}
J_{\mathrm{dc}}\left(V_{\mathrm{dc}}\right)= & J_{1 \rightarrow 2}+J_{2 \rightarrow 1}=J_{\mathrm{dc}}[\bar{\Phi} \exp (-A \sqrt{\bar{\Phi}}) \\
& \left.-\left(\bar{\Phi}+q V_{\mathrm{dc}}\right) \exp \left(-A \sqrt{\bar{\Phi}+q V_{\mathrm{dc}}}\right)\right]
\end{aligned}
$$

where $J_{\mathrm{dc}}=q / 2 \pi h(\beta \Delta s)^{2}, J_{1 \rightarrow 2}=J_{\mathrm{dc}}[\bar{\Phi} \exp (-A \sqrt{\bar{\Phi}})]$ is the current density flowing from metal 1 to metal 2 and $J_{2 \rightarrow 1}=-J_{\mathrm{dc}}\left(\bar{\Phi}+q V_{\mathrm{dc}}\right) \exp \left(-A \sqrt{\bar{\Phi}+q V_{\mathrm{dc}}}\right)$ is the current density flowing from metal 2 to metal 1 [see Fig. 1(b)], $A=$
$4 \sqrt{2 m_{0}} \pi \beta \Delta s / h, \beta$ is the correction factor defined in [34], $\bar{\Phi}$ and $\Delta s$ are the mean potential barrier height relative to Fermi level and barrier width for tunneling electrons, which must take into account the reduced potential barrier due to the image force [34]. We note that in such a nanostructure a transition to hopping or diffusive transport is difficult, since the field-induced breakdown occurs prior to reaching the bias necessary to bring about the change in mechanism, typical for quantum tunneling devices. It is known that when the applied bias is increased from zero to a high voltage, the carrier transport mechanism would transit from direct tunneling to field emission (Fowler-Nordheim tunneling [38]). See Supplemental Material [44] for the calculated I-V behavior of a silver-vacuum-silver nanojunction with a 1.2-nm air gap and a cross-sectional area $\pi a^{2}=\pi \cdot(5 \mathrm{~nm} \times 5 \mathrm{~nm})$; the work function of silver is $4.2 \mathrm{eV}$.

When a biased nanoantenna is illuminated by light, the applied electromagnetic field can have both dc component and ac optical harmonic components: $E=E_{\mathrm{dc}}+E_{\mathrm{op}}(t)=$ $\frac{V_{\mathrm{dc}}}{d}+\left(\frac{E_{\omega}}{2} e^{-i \omega t}+c . c\right)$, where $d$ is the size of air gap and $\omega$ is the radian frequency. The presence of optical fields across the MIM junction may adiabatically modulate the electron potential energy. It is, therefore, necessary to take into account the quantum mechanical phenomenon of photon-assisted tunneling $[25,35]$. According to the Tien-Gordon model $[35,36]$ for monochromatic external fields, new quantum well virtual states are introduced [see Fig. 1(b)], which are separated from the unperturbed ground state by $\pm n \hbar \omega$ with a state density proportional to $J_{n}\left(q E_{\omega} d / \hbar \omega\right)$, where $J_{n}(\cdot)$ is the $n$ th-order Bessel function and $\hbar \omega$ is the photon energy; here $n>0$ and $n<0$, respectively, represent the photon absorption and emission processes, which can be viewed as the creation of effective electron densities at energies $q E_{\omega} d \pm n \hbar \omega$. We should note that $V_{p h}=\hbar \omega / q$ is the photon voltage and $V_{\omega}=E_{\omega} d$ is the ac electric voltage across the nanogap related to the strength of the local electric fields $E_{\omega}$. Under the optical excitation, the dc photoelectric current $J_{\text {illum }}$ and the frequency-dependent tunneling currents for the $m$ th harmonic $J_{m \omega}$ can be expressed as $[35,36]$

$$
\begin{aligned}
J_{\text {illum }}= & \sum_{n=-\infty}^{\infty} J_{0}^{2}(\alpha) J_{\mathrm{dc}}\left(q V_{\mathrm{dc}}+n \hbar \omega\right) \\
J_{m \omega}= & \sum_{n=-\infty}^{\infty} J_{n}(\alpha)\left[J_{n+m}(\alpha)+J_{n-m}(\alpha)\right] e^{-i m \omega t} \\
& \times J_{\mathrm{dc}}\left(q V_{\mathrm{dc}}+n \hbar \omega\right),
\end{aligned}
$$

where $\alpha=q V_{\omega} / \hbar \omega$ is the ratio between the classic ac electric voltage and the quantum mechanical photon voltage. At near-infrared and visible frequencies, the photon voltage is usually greater than the ac electric voltage, i.e., $V_{\omega} \ll \hbar \omega / q$. The summation in (2) can be approximated by extending to the lowest order $(n=0$ and \pm 1$)$ [39], and the dc current under illumination is given by

$$
\begin{aligned}
J_{\text {illum }}= & J_{\mathrm{dc}}\left(V_{\mathrm{dc}}\right)+\Delta J_{\mathrm{dc}}\left(V_{\mathrm{dc}}\right) \\
\approx & J_{\mathrm{dc}}\left(V_{\mathrm{dc}}\right)+\left(\frac{q d}{2 \hbar \omega}\right)^{2}\left[J_{\mathrm{dc}}\left(V_{\mathrm{dc}}+\hbar \omega / q\right)\right. \\
& \left.-2 J_{\mathrm{dc}}\left(V_{\mathrm{dc}}\right)+J_{\mathrm{dc}}\left(V_{\mathrm{dc}}-\hbar \omega / q\right)\right],
\end{aligned}
$$


where the second term is related to the nonlinear effect of optical rectification [39]. The in-phase, ac-driven current density at the fundamental frequency $(m=1)$ can be obtained as

$$
\begin{aligned}
J_{\omega}= & J_{\omega}^{(1)}+J_{\omega}^{(3)} \\
\approx & \frac{q d}{\hbar \omega} \frac{J_{\mathrm{dc}}\left(V_{\mathrm{dc}}+\hbar \omega / q\right)-J_{\mathrm{dc}}\left(V_{\mathrm{dc}}-\hbar \omega / q\right)}{2} E_{\omega} e^{-i \omega t} \\
& +\left(\frac{q d}{2 \hbar \omega}\right)^{3}\left[\begin{array}{l}
J_{\mathrm{dc}}\left(V_{\mathrm{dc}}+2 \hbar \omega / q\right)-J_{\mathrm{dc}}\left(V_{\mathrm{dc}}-2 \hbar \omega / q\right) \\
-J_{\mathrm{dc}}\left(V_{\mathrm{dc}}+\hbar \omega / q\right)+J_{\mathrm{dc}}\left(V_{\mathrm{dc}}-\hbar \omega / q\right)
\end{array}\right] \\
& \times\left|E_{\omega}\right|^{2} E_{\omega} e^{-i \omega t} .
\end{aligned}
$$

The first term in (3) is related to a real, linear conductivity

$$
\sigma_{\omega}^{(1)}(\omega)=\frac{q d}{\hbar \omega} \frac{J_{\mathrm{dc}}\left(V_{\mathrm{dc}}+\hbar \omega / q\right)-J_{\mathrm{dc}}\left(V_{\mathrm{dc}}-\hbar \omega / q\right)}{2},
$$

which is associated with the power dissipation in the nanojuction. The second term in (3) is related to a real, third-order nonlinear conductivity

$$
\begin{aligned}
\sigma_{\omega}^{(3)} & \equiv \sigma^{(3)}(\omega ; \omega, \omega,-\omega) \\
& =\left(\frac{q d}{2 \hbar \omega}\right)^{3}\left[\begin{array}{l}
J_{\mathrm{dc}}\left(V_{\mathrm{dc}}+2 \hbar \omega / q\right)-J_{\mathrm{dc}}\left(V_{\mathrm{dc}}-2 \hbar \omega / q\right) \\
-J_{\mathrm{dc}}\left(V_{\mathrm{dc}}+\hbar \omega / q\right)+J_{\mathrm{dc}}\left(V_{\mathrm{dc}}-\hbar \omega / q\right)
\end{array}\right],
\end{aligned}
$$

which is associated with the two-photon absorption [39] and $J_{\omega}^{(3)}$ is dependent on the illumination intensity $\left(J_{\omega}^{(3)} \propto\left|E_{\omega}\right|^{2}\right)$. These linear and nonlinear conductivities in (4) and (5) are indeed important in the near-infrared and visible regimes, where the effective field strength yielded by photon $\hbar \omega / q d$ has a large value compared to the electric fields strength $E_{\omega}$ resulting from the pulsed laser beam. Hence, these quantum conductivities may contribute relevant resistive responses, which are frequency-dependent and can be controlled by the $\mathrm{dc}$ bias $\left(\sigma_{\omega}^{(1)}\right.$ and $\left.\sigma_{\omega}^{(3)}\right)$ or by the intensity of illuminating laser $\left(\sigma_{\omega}^{(3)}\right)$. In $(2)$, there are also other higher order conductivities, such as $\sigma_{3 \omega}^{(3)} \equiv \sigma^{(3)}(3 \omega ; \omega, \omega, \omega)$ associated with the thirdharmonic generation and four-wave mixing [40,41,45-47], which will be observed at high illumination intensity. The rectification and second-harmonic generation (SHG), related to the second-order conductivities $\sigma_{0}^{(2)} \equiv \sigma^{(2)}(0 ; \omega,-\omega)$ and $\sigma_{2 \omega}^{(2)} \equiv \sigma^{(2)}(2 \omega ; \omega, \omega)$, will exist in the asymmetry tunneling junction made by applying a dc bias or by designing nanodipole arms with asymmetric geometry or different metal work functions [48]. Recently, Haus et al. [40] and Scalora et al. [45] have theoretically demonstrated that these highorder quantum conductivities originating from the quantum gap of metallic nanostructures can be boosted by several orders of magnitude the amplitudes of the harmonic signals arising from the intrinsic nonlinearities of metals.

Figure 1(d) shows the optical nanocircuit model of the dipole nanoantenna in the receiving mode $[5,32,33]$. When incident light impinges upon the nanoantenna, it induces an open-circuit voltage $V_{o c}$ in the Thevemin's equivalent circuit: $V_{o c}=E_{\text {inc }} L_{\text {eff }}$, where $E_{\text {inc }}$ is the electric field amplitude of light, $L_{\text {eff }}$ is the effective length of nanoantenna given by $L_{\text {eff }}=\int_{-L / 2}^{L / 2} I\left(z^{\prime}\right) d z^{\prime} / I_{0}, \quad I_{0}$ is the imposed current at the middle of antenna, and $L$ is the length of nanoantenna [5,32].
Due to the small gap dimensions and the continuity of the displacement current flowing along the nanodipole, the electric field at the gap is uniform across the load and polarized along the dipole axis. Therefore, the air-load impedance is given by $[5,32,33]$

$$
Z_{\text {load }}=-\frac{1}{i \omega C_{g}}
$$

where $C_{g}=\varepsilon_{0} \frac{\pi a^{2}}{d}$ is the classical geometry-yielded capacitance, $a$ is the wire radius of nanodipole, and $\varepsilon_{0}$ is the free space permittivity. In the quantum regime, the quantum conductivity $\sigma_{\omega}^{(1)}(\omega)$ will give rise to a resistance in parallel to the classic capacitance $C_{g}$ as

$$
R_{Q}^{(1)}\left(\omega, V_{\mathrm{dc}}\right)=\frac{1}{\sigma_{\omega}^{(1)}\left(\omega, V_{\mathrm{dc}}\right)} \frac{d}{\pi a^{2}},
$$

which is responsible for the power dissipation. When the quantum mechanical effect is considered, the air-gap impedance becomes

$$
Z_{\text {load }}=\left[\left(R_{Q}^{(1)}\right)^{-1}-i \omega C_{g}\right]^{-1} .
$$

Intuitively, if the gap is large enough, the quantum conductivity induced by tunneling currents becomes so small that $Z_{\text {load }}$ approaches the classical limit in (6). When the intensity of illumination is high, a nonlinear quantum resistance $\sigma_{\omega}^{(3)}$ also comes into the load resistance [see Fig. 1(d)] in the nanocircuit model as

$$
R_{Q}^{(3)}\left(\omega, V_{\mathrm{dc}},\left|E_{g}\right|^{2}\right)=\frac{1}{\sigma_{\omega}^{(3)}\left(\omega, V_{\mathrm{dc}}\right)\left|E_{g}\right|^{2}} \frac{d}{\pi a^{2}},
$$

which depends on the optical power intensity inside the nanogap $\propto\left|E_{g}\right|^{2}$. As has been well-known from the plasmonic features of nanoantennas, $E_{g}$ can be orders of magnitude greater than that of the impinging light $E_{\text {inc }}$. Hence, the nonlinear absorption related to $R_{Q}^{(3)}$ could occur even at a relatively low illumination intensity. In the nanocircuit model, there is also an intrinsic dipole impedance $Z_{\text {dip }}=$ $R_{\text {dip }}-i X_{\text {dip }}$, where $R_{\text {dip }}=R_{\text {rad }}+R_{\text {loss }}, R_{\text {rad }}$ is the radiation resistance, responsible for the power loss by radiation (or scattering in the receiving mode), $R_{\text {loss }}$ is the resistance, responsible for the plasmon damping, and $X_{\text {dip }}$ is the nanoantenna reactance, responsible for the near-field stored energy. From the nanocircuit model, the voltage across the air gap $V_{\text {gap }}=V_{o c} Z_{\text {load }} /\left(Z_{\text {load }}+Z_{\text {dip }}\right)$, and the uniform electric field inside the gap is obtained as $E_{\text {gap }}=V_{\text {gap }} / d$. [32]. The nanoantenna resonance is achieved when $\operatorname{Im}\left[Z_{\text {load }}\right]=-X_{\text {dip }}$, resulting in the maximum enhancement of optical field at the gap. The quantum resistances may provide tremendous advantages in the optical regime, as the resistive properties of small nanoloads may be drastically enhanced and controlled with relatively low impinging optical intensities and applied bias. Here, we should note that the high-order harmonics will be shorted by the nanoantenna capacitance and therefore be suppressed. Indeed, when the nanoantenna is dc biased, the second-order quantum conductivity associated with the rectification and the SHG may be induced due to the asymmetric potential barrier in the MIM junction. As the harmonic losses are suppressed by the nanoantenna (i.e., the second and third harmonics are away from the high-order resonances 
of nanoantenna), the incident power is equal to the sum of incoming ac power across the MIM junction $1 / 2 I_{\omega} V_{o c}$, dc power dissipated $-\left|V_{\mathrm{dc}} \Delta I_{\mathrm{dc}}\right|$ in the MIM junction, and the rectified dc power available at the load $V_{\mathrm{dc}} \Delta I_{\mathrm{dc}}$ (where $\Delta I_{\mathrm{dc}}$ is the incremental current due to the illumination). In the case of no external load, the dc current is zero, and all input power is dissipated in the MIM junction as ac optical losses [26,27]. On the other hand, for the solar energy rectification and photodetection applications, an optimal load resistance is needed to more efficiently deliver the generated dc power to the load [25-27].

In the following, we will discuss how tunability and switching effects associated with the relevant quantum conductivities induced in the MIM nanojunction may be employed to realize electronically and optically tunable optical nanoantennas and metamaterials. Consider first an individual nanodipole shown in Fig. 1(a), with a diameter $2 a=10 \mathrm{~nm}$, length $L=90 \mathrm{~nm}$, and gap $d=1.2 \mathrm{~nm}$. This nanoantenna is illuminated by a plane wave propagating in the $z$ axis, with electric field polarized parallel to the dipole arms ( $x$ axis). Here, the nanoantenna's dipole arms are silver nanorods, with the relative permittivity following the Drude-type dispersion model $\varepsilon_{\text {Ag }}=$ $\varepsilon_{\infty}-\omega_{p}^{2} /[\omega(\omega+i \gamma)]$, with $\omega_{p} / 2 \pi=2175 \mathrm{THz}, \gamma / 2 \pi=$ $4.35 \mathrm{THz}$, and $\varepsilon_{\infty}=5$ [49]. For simplicity, we ignore the third-order nonlinearity of metal. Assuming a linear optical regime, such nanoantenna resonates at a frequency of $f_{0}=$ $350 \mathrm{THz}$, providing the extreme light localization and field enhancement $\left(\left|E_{g} / E_{\text {inc }}\right|=1080\right)$. The intrinsic impedance of this nanoantenna is reported in the Supplemental Material [44]. Figure 2(a) plots the linear quantum resistance $R_{Q}^{(1)}$ versus frequency of light for different dc bias voltages; here, we also present the classic ac resistance $R_{c}=\left(\partial I_{\mathrm{dc}} / \partial V_{\mathrm{dc}}\right)^{-1}$ and $I_{\mathrm{dc}}=J_{\mathrm{dc}} \times \pi a^{2}$ for comparison. It can be seen from Fig. 2(a) that the quantum resistance can differ greatly from the classic resistance at high frequencies, i.e., near-infrared and visible regimes. In addition, the value of $R_{Q}^{(1)}$ decreases with increasing the bias voltage, due to the potential barrier lowering that facilitates the electron tunneling through the MIM nanojunction. At the nanoantenna resonance, if the value of $R_{Q}^{(1)}$ is close to the order of $R_{\text {dip }}$, achievable with high bias voltages or aggressively scaling the gap, the power dissipation in the resistive nanoload (MIM nanojunction) increases significantly,
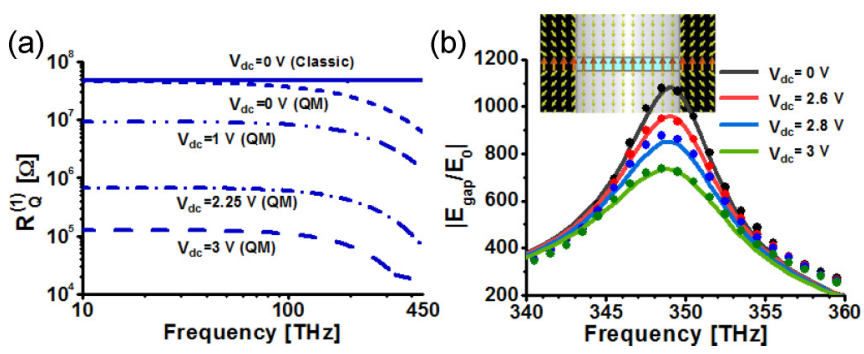

FIG. 2. (Color online) (a) Quantum resistance versus frequency for different bias conditions. (b) Optical field enhancement inside the nanogap versus frequency for a nanoantenna with $L=90 \mathrm{~nm}, 2 a=$ $10 \mathrm{~nm}$, and $d=1.2 \mathrm{~nm}$, under different bias conditions; here solid lines and dots represent results obtained from the nanocircuit model in Fig. 1(d) and the full-wave simulation, respectively. Inset of Fig. 2(b) shows the electric field distributions in the near-field air gap.
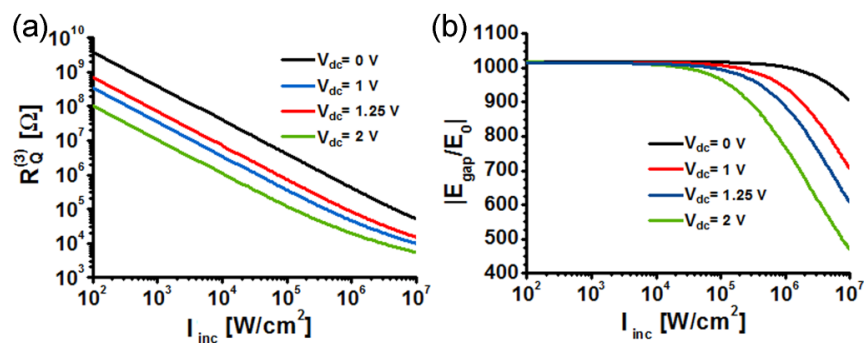

FIG. 3. (Color online) (a) Third-order nonlinear quantum conductivity $R_{Q}^{(3)}$ versus illumination intensity for the nanoantenna in Fig. 2 at the resonance frequency $f_{0}=350 \mathrm{THz}$, under different dc bias conditions. (b) is similar to (a), but plotting the corresponding optical field enhancement.

thereby deteriorating the optical field enhancement effect. Figure 2(b) plots the maximum field enhancement $\left|E_{g} / E_{\text {inc }}\right|$ versus frequency for different bias voltages; the solid lines and dots represent the results obtained from the nanocircuit model in Fig. 1(d) and full-wave simulations, based on the finite-integration technique [50], respectively. It is evident that the nanocircuit model can quantitatively predict the field enhancement around the resonance frequency, showing excellent agreement with numerical results. As can be seen from Fig. 2(b), the field enhancement decreases with increasing the dc bias voltage, which reduces the loaded resistance $R_{Q}^{(1)}$ and therefore increases the overall power dissipation. In the zero-bias case, the quantum mechanical effect is almost negligible, and the maximum field enhancement is comparable to that obtained from the classical model. When the gap is further reduced to subnanometer, the quantum resistance $R_{Q}^{(1)}$ will be dramatically lowered. This quantum resistance essentially places a fundamental limit on the maximum field enhancement when the air gap is downscaled [39].

At high illumination intensity, the strongly localized optical fields may greatly enhance possible nonlinear optical effects, i.e., $R_{Q}^{(3)}$, in the load region. Figure 3 (a) plots $R_{Q}^{(3)}$ versus the intensity of impinging light at the resonance frequency $f_{0}=350 \mathrm{THz}$ for different bias conditions. It is strikingly seen that the values of $R_{Q}^{(3)}$ decrease logarithmically with increasing the illumination intensity. Further, $R_{Q}^{(3)}$ is also reduced through the electrostatic biasing, similar to Fig. 2(a), because of the enhanced photon-assisted tunneling. Figure 3(b) plots the field enhancement versus illumination intensity at $f_{0}=350 \mathrm{THz}$, showing a notable reduction in optical field enhancement at high optical intensity. This arises from the fact that the third-order nonlinear quantum conductivity $R_{Q}^{(3)}$ is proportional to the local light intensity $\left|E_{g}\right|^{2}$ at the gap. At high illumination intensity, implying a nonlinear optical regime, $R_{Q}^{(3)}$ can sensibly affect the field enhancement in the gap region, particularly under a dc biased field.

For a single nanoantenna, the far-field scattering reaches its maximum at the resonance condition [5]. The scattering power can be evaluated within the nanocircuit model in Fig. 1(d) as

$$
P_{\text {sca }}=\frac{1}{2}\left|\frac{V_{o c}}{Z_{\text {dip }}+Z_{\text {load }}}\right|^{2} R_{\text {rad }},
$$




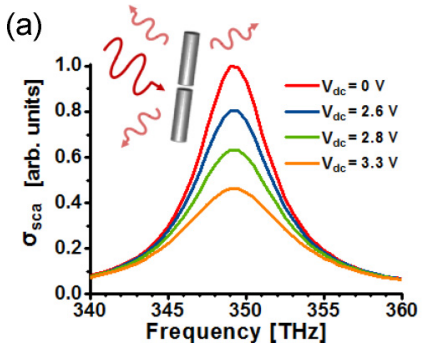

(b)

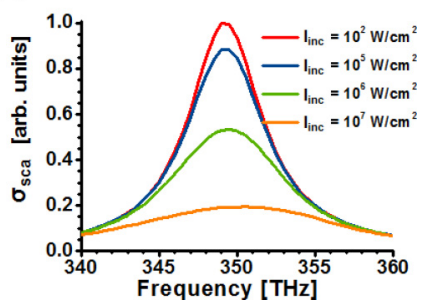

FIG. 4. (Color online) (a) Scattering cross section versus frequency for the nanoantenna in Fig. 3(a); we assume a linear optical regime, i.e., low illumination intensity, and vary the bias condition, and (b) is similar to (a), but for the nonlinear optical regime, i.e.. fairly high illumination intensity, varying the illumination intensity; the dc bias is fixed $V_{\mathrm{dc}}=2 \mathrm{~V}$.

where $R_{\text {rad }}$ can be evaluated from the simulated radiation efficiency $\eta=R_{\mathrm{rad}} /\left(R_{\mathrm{rad}}+R_{\mathrm{loss}}\right)$ and the intrinsic dipole impedance; here, $\eta \approx 20 \%$ around the operating frequency was obtained from the full-wave simulation. The scattering cross section, as the ratio of scattering power to intensity of impinging light $I_{\text {inc }}\left(\sigma_{\text {sca }}=P_{\text {sca }} / I_{\text {inc }}\right)$, can indicate the degree to which an object scatters. It is obvious from (7) and (8) how the applied dc bias and the impinging light intensity can tune the $R_{O}^{(1)}$ and $R_{O}^{(3)}$ over a wide range in the visible regime, which in turn influence $\operatorname{Re}\left[Z_{\text {load }}\right]$. Assuming a low illumination intensity (in the linear regime), Fig. 4(a) reports the scattering cross section versus frequency for an individual quantum nanoantenna. It is clearly seen that the scattering power decreases with increasing the dc bias in light of the increased power dissipations caused by $R_{Q}^{(1)}$. Figure $4(\mathrm{~b})$ is similar to Fig. 4(a), but for the nonlinear case operated with fairly high optical intensities; here, the applied bias $V_{\mathrm{dc}}=2 \mathrm{~V}$ and the illumination intensity $I_{\text {inc }}$ is varied from 10 to $10^{7} \mathrm{~W} / \mathrm{cm}^{2}$. Since $R_{Q}^{(3)}$ is inversely proportional to the light intensity, high illumination intensity may result in an increased amount of power absorption from the impinging light, thus yielding smaller scattering cross section. It is seen from Fig. 4(b) that the scattering cross section can be switched from low to high by changing the intensity of impinging light. The intriguing properties in Figs. 4(a) and 4(b) may potentially realize a switchable and modulatable nanoantenna with the voltage- or intensity-controlled scattering properties. Possible applications include the optical nanologics or nanoswitches [19].

The plasmonic properties of the nanodipole also shortens the effective guided wavelength along the antenna [4], implying a resonant length $L \approx \lambda_{0} / 9.5$, where $\lambda_{0}$ is the free-space wavelength of light. The long-wavelength resonance may be employed to build optical metasurfaces (metamaterials) consisting of quasihomogeneous planar (volumetric) arrays of such nonlinear elements. Consider the metasurface shown in the inset of Fig. 5(a); the impinging light is a normal incident TE wave, with electric field polarized parallel to the dipole axis of nanoantennas. The collective behavior of induced dipole moments in nanoantennas generates a homogeneous, thin sheet of surface current that radiates symmetrically on both sides. The total averaged polarization related to the local
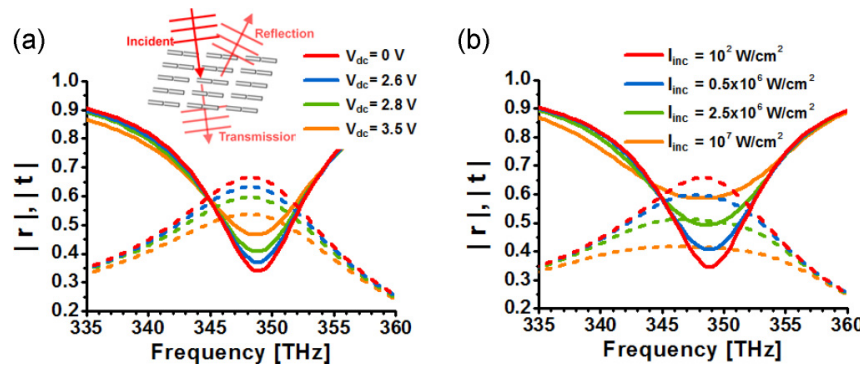

FIG. 5. (Color online) (a) Transmission (solid line) and reflection (dashed line) coefficients versus frequency for the optical metasurface in Fig. 1(c) at low illumination intensity, varying the bias conditions, and (b) is similar to (a), but for different levels of illumination intensities, under a fixed dc bias $V_{\mathrm{dc}}=2 \mathrm{~V}$.

field $\mathbf{E}_{\mathrm{loc}}=E_{\mathrm{loc}} \hat{\boldsymbol{x}}$ is responsible to the component $\alpha_{x x}$ of the polarizability tensor. Considering the full coupling among the whole array of interacting nanoantennas, the dipole moment induced on the nanoparticle is given by [51]

$$
\begin{aligned}
\mathbf{P}_{N^{\prime} x N^{\prime} y} & =\alpha_{x x} \mathbf{E}_{\mathrm{loc}} \\
& =\alpha_{x x}\left[\sum_{\left(N_{x}, N_{y}\right) \neq\left(N^{\prime} x, N^{\prime} y\right)} \mathbf{G}\left(\mathbf{r}_{N_{x} N_{y}}-\mathbf{r}_{N^{\prime} x, N^{\prime} y}\right) \cdot \mathbf{P}_{N^{\prime} x, N^{\prime} y}\right],
\end{aligned}
$$

where $N_{x}$ and $N_{y}$ are positive or negative integers that relate the position of nanoantenna and periods $a_{x}$ and $a_{y}$ by $x=N_{x} a_{x}$ and $y=N_{y} a_{y}$, and $\mathbf{G}\left(\mathbf{r}_{N_{x} N_{y}}\right)=\left(\nabla \nabla+k^{2} \overline{\overline{\mathbf{I}}}\right) e^{i k_{0} r} / 4 \pi r$ is the dyadic Green's function in free space. After some algebraic manipulation, we may write (11) as

$$
\mathbf{P}_{00}=P_{00} \hat{x}=\frac{\mathbf{E}_{\text {inc }}}{\alpha_{x x}^{-1}-C},
$$

where $C=\sum_{\left(N_{x}, N_{y}\right) \neq(0,0)} \mathbf{G}\left(\mathbf{r}_{N_{x} N_{y}}\right) \cdot \hat{\boldsymbol{x}} \cdot \hat{\boldsymbol{x}}$ is the interaction constant that relates the fields induced by the infinite array of dipoles around the unit cell under consideration to the local field at the origin. The averaged surface current density on metasurface can be expressed as

$$
\mathbf{J}_{\mathrm{av}}=J_{\mathrm{av}} \hat{\boldsymbol{x}}=\frac{-i \omega \mathbf{P}_{00}}{a_{x} a_{y}}=\frac{-i \omega E_{\mathrm{inc}}}{a_{x} a_{y}\left(\alpha_{x x}^{-1}-C\right)} \hat{\boldsymbol{x}} .
$$

This is an accurate description of the electromagnetic properties of the array, as long as the periods are small enough to ensure that only one Floquet harmonic affects the far-field radiation from the surface. When the metasurface is illuminated by light, the induced surface current produces a reflected wave with the electric fields given by $\mathbf{E}_{\mathbf{r}}=-\left(\omega \mu_{0} / 2 k_{0}\right) J_{\mathrm{av}} e^{-i k_{0} z} \hat{\boldsymbol{x}}$, and the transmitted field can be similarly derived as $\mathbf{E}_{\mathrm{t}}=\mathbf{E}_{\mathrm{inc}}-\left(\omega \mu_{0} / 2 k_{0}\right) J_{\mathrm{av}} e^{i k_{0} z} \hat{\boldsymbol{x}}$. As a result, the reflection and transmission coefficients can be calculated as

$$
\begin{aligned}
r & =\frac{i \omega^{2} \mu_{0}}{2 k_{0} a_{x} a_{y}\left(\alpha_{x x}^{-1}-C\right)} \\
t & =1+r .
\end{aligned}
$$


The average sheet impedance of metasurface, as the ratio of the local electric field to the surface current density, reads

$$
Z_{s}=\frac{E_{\text {inc }}}{J_{\mathrm{av}}}-\frac{\eta_{0}}{2},
$$

where $\eta_{0}$ is the free space impedance. The polarizability of a subwavelength dipole antenna, responsible for the scattering properties, has an explicit expression as a function of impedances $Z_{\text {load }}$ and $Z_{\text {dip }}$ [52]:

$$
\alpha_{x x}=i \frac{L^{2}}{12 \omega Z_{\mathrm{dip}}}\left(\frac{Z_{\mathrm{dip}}+Z_{\mathrm{load}}}{4 Z_{\mathrm{dip}}+Z_{\mathrm{load}}}\right) .
$$

From (14), we know that the metasurface resonance arises under the condition

$$
\operatorname{Re}\left[\left(\alpha_{x x}\left(Z_{\text {load }}\right)\right)^{-1}\right]=\operatorname{Re}[C] .
$$

Assuming a linear optical regime, Fig. 5(a) reports the transmission and reflection coefficients calculated using (14) for the metasurface formed by nanoantennas in Fig. 4; here, $a_{x}=a_{y}=1.8 \mathrm{~L}$. It is observed that the transmission and reflection magnitudes can be tuned by applying a bias voltage across the transparent graphene electrodes contacting the metasurface, as shown in Fig. 1(c). Since $\operatorname{Im}\left[\alpha_{x x}\right]$ associated with the power balance [51] is a function of $R_{Q}^{(1)}$, as described in (16), the minimum transmission and maximum reflection can be modulated by varying the load resistance $R_{Q}^{(1)}$ (and also $R_{Q}^{(3)}$ at high illumination intensity). As expected, when the bias voltage is increased, the operating bandwidth is increased slightly due to the reduction of the quality factor ( $Q$ factor), and the transmission dip increases due to the increased surface resistance of this metasurface. Figure 5(b) is similar to Fig. 5(a), but for the nonlinear optical regime, under a constant dc bias $V_{\mathrm{dc}}=2 \mathrm{~V}$ and different illumination levels. It is seen that the depth of the transmission dip and the corresponding reflection peak are reduced by increasing the intensity of impinging light, directly attributed to the increased importance of the nonlinear resistance $R_{Q}^{(3)}$ associated with the two-photon absorption. In this case, the effect of nonlinear absorption becomes relevant when the impinging light intensity is greater than $10 \mathrm{~K} \mathrm{~W} / \mathrm{cm}^{2}$. Note that the optical properties of this metasurface can be tuned by varying the applied bias or the illumination intensity, thanks to the quantum-induced linear and nonlinear resistances in the gap region. We note that one can build a three-dimensional optical metamaterial stacking up multiple planar nanoantenna arrays along the propagation direction in order to enhance the modulation depth of transmission and reflection and to achieve the extreme effective material properties. Moreover, the quantum conductivity can be boosted by reducing the barrier height of tunneling nanojucntion, i.e., loading dielectric nanoparticles with high electron affinity or using metals with lower work functions. This will remarkably enhance the photon-assisted tunneling effect induced in the nanojunction built by metallic nanoantennas. Possible applications of the nonlinear quantum conductivities also include a high harmonic generation, rectification, and wave mixing [39,41,45-47], which can be utilized to realize various nanophotonic sources, sensors, and detectors [25-27], as well as energy harvesting nanodevices [25-27], (i.e., optical rectennas). We should also note that when a nanoantenna is loaded with nonlinear dielectrics, the resonance frequency is also shiftable due to the electro-optic effect. The optical index modulation may provide an extra design flexibility in terms of tuning and modulating the optical responses. Therefore, a quantum nanoantenna may potentially achieve both phase and amplitude modulation. We notice that the work function or structural variations, e.g., the gap size of nanoantenna due to the fabrication inaccuracy, may sensitively cause the change in quantum conductivities. The conductivity fluctuations, however, could be compensated by the applied bias, giving the consistent loaded quantum resistance and optical properties.

Finally, we note that quantum nanoantennas with switchable scattering properties may have many interesting applications. One of its potential applications is to produce the dynamic holography process at the nanoscale [16-18], which is achieved by tailoring the local surface impedance or scattering properties to make an amplitude-modulation hologram [53]. Optical holography involves producing an interference pattern, the so-called hologram, using two waves. When the reference wave illuminates the hologram, it is scattered by the recorded interference pattern to produce a copy of the original object wave. For conventional holograms made of passive diffraction gratings or structured surfaces, once the hologram is recorded, the wave front of the recovered object wave may not be changed. Nonetheless, the variable quantum conductivity of the MIM nanojunction together with the sensitive optical response of the plasmonic nanoantenna unveils the unique possibility to tailor the local surface impedance (or reflection and transmission magnitudes) of the metasurface, necessary for achieving the amplitude-modulation digital holography. Figure 6(a) illustrates an example for a planar lens designed by the holography method; here, the biased and unbiased nanoantennas are, respectively, represented by the red and blue colors, making an active and tunable nanoscale hologram. Depending on the biasing setup and the incident optical power, a normal incident plane wave with the electric field polarized parallel to nanoantennas can be totally reflected or be transferred into specific wave fronts. Assume that, we would like to have a focused light sheet, the interference between fields associated with the incident plane wave (i.e., reference wave $e^{i \mathbf{k} \cdot\left(\mathbf{r}-\mathbf{r}^{\prime}\right)}$ ) and the scattered wave (i.e., object wave $e^{i \mathbf{k}\left(\mathbf{r}-\mathbf{r}^{\prime}\right)} /\left(\mathbf{r}-\mathbf{r}^{\prime}\right)$, which can be considered as an infinite line source) on the nanoantenna metasurface will define a hologram. For the amplitude-modulation holography, the digitized modulation pattern can be derived from the coherent superposition of optical wave fields as an intensity function [53-55]

$$
I(\mathbf{r})=\left|\frac{e^{i|\mathbf{k}|\left|\mathbf{r}-\mathbf{r}^{\prime}\right|}}{\left|\mathbf{r}-\mathbf{r}^{\prime}\right|}+e^{i \mathbf{k} \cdot\left(\mathbf{r}-\mathbf{r}^{\prime}\right)}\right|^{2},
$$

where $I(\mathbf{r})$ represents the interference pattern, $\mathbf{r}^{\prime}$ is the position of the infinite line source, and $\mathbf{k}$ is the wave vector of radiation. Here, we assume the infinite line source located $2 \lambda_{0}$ away from the metasurface and a normal incident plane wave, as shown in Fig. 6(a). Figure 6(b) shows the contours of electric field amplitude for the metasurface formed by a 2-D array of nanoantennas in Figs. 4 and 5, with $a_{x}=a_{y}=0.7 \mathrm{~L}$, under the zero-bias condition and the incident optical intensity $I_{i n c}=25 \mathrm{MW} / \mathrm{cm}^{2}$. Due to the quenching effect, the resonance frequency for this dense nanoantenna array is redshifted 

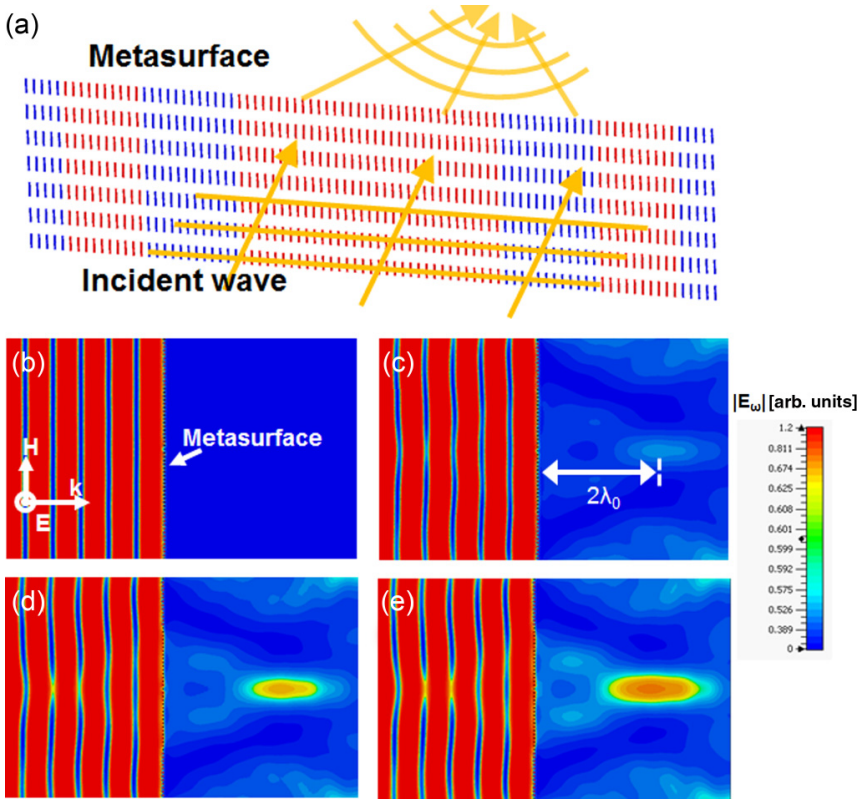

FIG. 6. (Color online) (a) Schematic of nanoholography using a metasurface formed by quantum nanoantennas; the blue-colored nanoantennas are unbiased and the red-colored nanoantennas are biased with $V_{\mathrm{dc}}=2.25 \mathrm{~V}$. When a normal incident plane wave impinges such hologram, a focused light sheet will be produced. (b) Contours of the amplitude of electric fields (top view) in the zero-bias condition, at the illumination intensity $I_{\text {inc }}=25 \mathrm{MW} / \mathrm{cm}^{2}$. (c)-(e) are similar to (b), but with nanoantennas biased to achieve the digital hologram in (a), for different levels of illumination intensities: (c) $I_{\mathrm{inc}}=1 \mathrm{MW} / \mathrm{cm}^{2}$, (d) $I_{\mathrm{inc}}=10 \mathrm{MW} / \mathrm{cm}^{2}$, and (e) $I_{\text {inc }}=25 \mathrm{MW} / \mathrm{cm}^{2}$.

to $320 \mathrm{THz}$ [19]. In the zero-bias condition, the effect of quantum conductance is weak and the standing-wave patterns due to high reflection at the metasurface's resonance are observed. Next, we adopt the hologram in Fig. 6(a), where the blue- and red-colored nanoantennas are unbiased and biased to $V_{\mathrm{dc}}=2.25 \mathrm{~V}$, respectively. Figures $6(\mathrm{c})-6(\mathrm{e})$ show the contours of electric field amplitude for the same metasurface in Fig. $6\left(\right.$ b) at $f_{0}=320 \mathrm{THz}$, but with a dc bias $V_{\mathrm{dc}}=$ $2.25 \mathrm{~V}$ for those red-colored nanoantennas, under different illumination intensities: Fig. 6(c) $I_{\text {inc }}=5 \mathrm{MW} / \mathrm{cm}^{2} ;$ Fig. 6(d) $I_{\text {inc }}=10 \mathrm{MW} / \mathrm{cm}^{2}$; and Fig. $6(\mathrm{e}) I_{\text {inc }}=25 \mathrm{MW} / \mathrm{cm}^{2}$. It is surprisingly seen from Figs. 6(b)-6(e) that the object image (i.e., the focused light sheet) appears by biasing the nanoantennas based on the analytical modulation pattern obtained in (18) and by increasing the illumination intensity, which, in turn, enhances the modulation depth of hologram. As known from Fig. 5, the magnitude of (local) transmission for a unit cell of metasurface can be tailored by varying the dc bias and the illumination intensity. Therefore, the amplitude-modulation hologram can be synthesized with the controllable modulation period and depth, somehow similar to those conventional holographic gratings, but using a dynamically modulatable nanoscale monolayer. In Figs. 6(b)-6(e), the effect of standing wave is still observed in the direction of the counterpropagation, due to the partial reflection of the metasurface. The electronically/optically tunable quantum nanoantennas may pave a viable way to realize the dynamic holography with the reconstructed object wave being spatially reconfigurable in real time.

To conclude, we have presented the tunable and modulable optical radiators and metasurfaces formed by individual and arrayed quantum nanoantennas with a submicroscopic feed gap. We have shown that an external dc bias may significantly enhance the photon-assisted tunneling of plamsons in metallic nanorods, yielding a finite quantum conductivity that dynamically regulates the resistive responses to modulate the level of scattering, reflection, and transmission, and diffraction of light. On the other hand, the third-order nonlinear quantum conductivity may enable an all-optical self-tuning mechanism, by controlling the two-photon absorption level with illumination intensity. We envisage here a number of intriguing applications of quantum-nanoantennas, including tunable metamaterials and metasurfaces, nanoholography, nanophotonic switches, and voltage- and light-controlled attenuators, filters, and modulators.

The authors thank Dr. Andrea Alù at the University of Texas at Austin for fruitful discussions in plasmonic metasurfaces.
[1] K. B. Crozier, A. Sundaramurthy, G. S. Kino, and C. F. Quate, J. Appl. Phys. 94, 4632 (2003).

[2] P. J. Schuck, D. P. Fromm, A. Sundaramurthy, G. S. Kino, and W. E. Moerner, Phys. Rev. Lett. 94, 017402 (2005).

[3] P. Muhlschlegel, H. J. Eisler, O. J. F. Martin, B. Hecht, and D. W. Pohl, Science 308, 1607 (2005).

[4] L. Novotny, Phys. Rev. Lett. 98, 266802 (2007).

[5] A. Alù and N. Engheta, Nat. Photonics 2, 307 (2008).

[6] J. A. Schuller, T. Taubner, and M. L. Brongersma, Nat. Photonics 3, 658 (2009).

[7] P. Biagioni, J. S. Huang, and B. Hecht, Rep. Prog. Phys. 75, 024402 (2012).

[8] S. Palomba, M. Danckwerts, and L. Novotny, J. Opt. A: Pure Appl. Opt. 11, 114030 (2009).
[9] L. Novotny and N. V. Hulst, Nat. Photonics 5, 83 (2011).

[10] N. Yu, P. Genevet, M. A. Kats, F. Aieta, J. P. Tetienne, F. Capasso, and Z. Gaburro, Science 334, 333 (2011).

[11] N. Yu, P. Genevet, F. Aieta, M. A. Kats, R. Blanchard, G. Aoust, J. P. Tetienne, Z. Gaburro, and F. Capasso, IEEE J. Sel. Top. Quantum Electron 19, 4700423 (2013).

[12] I. S. Maksymov, A. E. Miroshnichenko, and Y. S. Kivshar, Opt. Express 20, 8929 (2012).

[13] D. Dregely, R. Taubert, J. Dorfmüller, R. Vogelgesang, K. Kern, and H. Giessen, Nat. Commun. 2, 267 (2011).

[14] P. Y. Chen, M. Farhat, and A. Alù, Phys. Rev. Lett. 106, 105503 (2011).

[15] A. Kriesch, S. P. Burgos, D. Ploss, H. Pfeifer, H. A. Atwater, and U. Peschel, Nano Lett. 13, 4539 (2013). 
[16] N. Horiuchi, Nat. Photonics 8, 84 (2014).

[17] S. Larouche, Y. J. Tsai, T. Tyler, N. M. Jokerst, and D. R. Smith, Nat. Mater. 11, 450 (2012).

[18] X. Ni, A. V. Kildishev, and V. M. Shalaev, Nat. Commun. 4, 2807 (2013).

[19] P. Y. Chen and A. Alù, Phys. Rev. B, 82, 235405 (2010).

[20] C. Argyropoulos, P. Y. Chen, F. Monticone, G. D'Aguanno, and A. Alù. Phys. Rev. Lett. 108, 263905 (2012).

[21] Y. Zhao, M. A. Belkin, and A. Alù, Nat. Commun. 3, 870 (2012).

[22] J. Sun, E. Timurdogan, A. Yaccobi, E. S. Hosseini, and M. R. Watts, Nature (London) 493, 195 (2013).

[23] M. W. Knight, H. Sobhani, P. Nordlander, and N. J. Halas, Science 332, 702 (2011).

[24] A. Moreau, C. Ciraci, J. J. Mock, R. T. Hill, Q. Wang, B. J. Wiley, A. Chilkoti, and D. R. Smith, Nature (London) 492, 86 (2012)

[25] S. Grover and G. Moddel, IEEE J. Photovolt. 1, 78 (2011).

[26] N. M. Miskovsky, P. H. Cutler, A. Mayer, B. L. Weiss, B. Willis, T. E. Sullivan, and P. B. Lerner, J. Nanotechnol. 2012, 512379 (2012).

[27] S. Grover, S. Joshi, and G. Moddel, J. Phys. D 46, 135106 (2013).

[28] P. Y. Chen and A. Alù, Nano Lett. 11, 5514 (2011).

[29] P. Y. Chen, C. Argyropoulos, and A. Alù, Nanophotonics 1, 221 (2012).

[30] P. Y. Chen and A. Alù, Nanotechnology 24, 455202 (2013).

[31] N. Engheta, Science 317, 1698 (2007).

[32] A. Locatelli, C. D. Angelis, D. Modotto, S. Boscolo, F. Sacchetto, M. Midrio, A. D. Capobianco, F. M. Pigozzo, and C. G. Someda, Opt. Express 17, 16792 (2009).

[33] N. Liu, F. Wen, Y. Zhao, Y. Wang, P. Nordlander, N. J. Halas, and A. Alù, Nano Lett. 13, 142 (2013).

[34] J. G. Simmons, J. Appl. Phys. 34, 1793 (1963).

[35] P. K. Tien and J. P. Gordon, Phy. Rev. 129, 647 (1963).

[36] J. R. Truker, IEEE J. Quantum Electron. 15, 1234 (1979).

[37] J. R. Tucker and M. J. Feldman, Rev. Mod. Phys. 57, 1055 (1985).
[38] J. M. Beebe, B. Kim, J. W. Gadzuk, C. D. Frisbie, and J. G. Kushmerick, Phys. Rev. Lett. 97, 026801 (2006).

[39] J. W. Haus, D. de Ceglia, M. A. Vincenti, and M. Scalora, J. Opt. Soc. Am. B 31, 259 (2014).

[40] J. W. Haus, D. de Ceglia, M. A. Vincenti, and M. Scalora, J. Opt. Soc. Am. B 31, A13 (2014).

[41] G. Hajisalem, M. S. Nezami, and R. Gordon, Nano Lett. 14, 6651 (2014).

[42] J. Zuloaga, E. Prodan, and P. Nordlander, Nano Lett. 9, 887 (2009).

[43] D. C. Marinica, A. K. Kazansky, P. Nordlander, J. Aizpurua, and A. G. Borisov, Nano Lett. 12, 1333 (2012).

[44] See Supplemental Material at http://link.aps.org/supplemental/ 10.1103/PhysRevB.91.035426 for the calculated current (I)voltage $(\mathrm{V})$ behavior of a silver-vacuum-silver nanojunction and intrinsic impedance of the nanoantenna.

[45] M. Scalora, M. A. Vincenti, D. de Ceglia, and J. H. Haus, Phys. Rev. A 90, 013831 (2014).

[46] M. Danckwerts and L. Novotny, Phys. Rev. Lett. 98, 026104 (2007).

[47] A. Stolz, J. Berthelot, M. M. Mennemanteauil, G. C. des Francs, L. Markey, V. Meunier, and A. Bouhelier, Nano Lett. 14, 2330 (2014).

[48] R. W. Boyd, Nonlinear Optics,3rd ed. (Academic Press, San Diego, CA, 2008).

[49] P. B. Johnson and R. W. Christy, Phys. Rev. B 6, 4370 (1972).

[50] CST Microwave Studio, 2014, http://www.cst.com.

[51] A. Alù and N. Engheta, in Structured Surfaces as Optical Metamaterials, edited by A. A. Maradudin (Cambridge University Press, Cambridge, 2011).

[52] S. Tretyakov, Analytical Modeling in Applied Electromagnetics (Artech House, London, 2003).

[53] R. Collier, C. Burckhardt, and L. Lin, Optical Holography (Academic Press, New York, 1971).

[54] P. Y. Chen, M. Farhat, A. N. Askarpour, M. Tymchenko, and A. Alù, J. Opt. 16, 094008 (2014).

[55] B. H. Fong, J. S. Colburn, J. J. Ottusch, J. L. Visher, and D. F. Sievenpiper, IEEE Trans. Antennas Propag. 58, 3212 (2010). 